\title{
Desenvolvimento e formação profissional em medicina geral e de família na Alemanha
}

\author{
Development and professional qualification of general practice and family medicine in Germany \\ Desarrollo y formación profesional en medicina general y familiar en Alemania
}

Markus Herrmann. Institut für Allgemeinmedizin. markus.herrmann@med.ovgu.de

Ligia Giovanella. Escola Nacional de Saúde Pública (ENSP/FIOCRUZ). giovanel@ensp.fiocruz.br (Autora correspondente)

\section{Resumo}

Objetivo: 0 artigo objetiva analisar o desenvolvimento profissional em medicina geral e de família (MGF) na Alemanha e discutir seus desafios. Métodos: estudo de caso que contextualiza a atuação em MGF na estrutura do sistema de saúde alemão, identifica as características e os instrumentos de intervenção, descreve as exigências para a especialização em MGF e analisa o processo de qualificação da especialidade. Resultados: A atenção por médico generalista na Alemanha tem tradição, contudo a ausência de função de gatekeeper enfraquece sua posição no sistema. A MGF passou nas últimas décadas por transformações, deixando de ser prática fundamentada no empirismo do Hausarzt de estilo tradicional para se transformar em especialidade em bases acadêmicas que tem como requisito cinco anos de formação prática em serviços de medicina geral e em áreas específicas de medicina interna, cirurgia e pediatria nos moldes de residência, regulada pelas câmaras médicas estaduais. Novas exigências curriculares nos últimos anos levaram a intenso processo de academização, com a criação de departamentos de MGF em 20 das 36 faculdades públicas de medicina do país. Conclusões: A crescente profissionalização acadêmica de outros trabalhadores da saúde, que na Alemanha tradicionalmente eram carreiras não universitárias, requer competências dialógicas para cooperação entre os diversos profissionais e para tomada de decisão com 0 paciente. 0 desenvolvimento acadêmico da MGF fortifica a pesquisa e contribui para aprimorar a base institucional da MGF como ciência, possibilitando interação e colaboração mais arrojadas com outros ramos da medicina e a real valorização da MGF.

\section{Abstract}

Objective: This article aims to analyze the professional and faculty development of general practice and family medicine (GP/FM) in Germany and discuss its facing challenges. Methods: It is a case study identifying characteristics and potential intervention tools, describing training and qualification requirements in family medicine in Germany. Results: The traditional caring role of GP in Germany has a long history, but GP has no gatekeeper function, which weakens its position in the system. In the past decades, GP has undergone several transformations; it is no longer a practice based on the traditional "Hausarzt" style. It has become a medical specialty of primary care with more modern foundations; it requires five years of practical training in internal medicine, paediatrics, surgery and general medicine, and it is governed by the Physician Chambers. In undergraduate education, courses in General Practice are mandatory. In recent years, the new curriculum requirements have led to an intense process of academic development with the creation of General Practice departments in 20 of the 36 public medical schools in the country. Conclusions: The process of professionalization and faculty development in GP/FM as well as the expansion of undergraduate training in the specialty aim to enhance the appeal of GP/FM to young doctors. This development strengthens academic research on GP/FM, which contributes to enhancing the institutional basis of GP/FM as a science, allowing bolder interaction and collaboration with other branches of medicine and real appreciation of this subject (GP/FM).

\section{Resumen}

El artículo tiene como objetivo analizar el desarrollo profesional en medicina general y familiar (MG/MF) en Alemania y discutir sus desafíos. Métodos: estudio de caso que contextualiza las características y las herramientas de actuación y describe los requisitos, en términos de entrenamiento y cualificación, necesarios para participar de la MF en Alemania. Resultados: La atención del MG en Alemania tiene tradición, pero la ausencia de función de gatekeeper debilita su posición en el sistema. La MG se transformó en las últimas décadas, dejando ser práctica que se basa en el estilo tradicional del „Hausarzt“. Se ha convertido en la especialidad académica de cuidado primario con fundamentos modernos que requiere cinco años de formación práctica en servicio en medicina general, pediatría, cirugía y medicina general, regulada por las Cámaras Médicas. En la educación de grado, los cursos de Medicina General son obligatorios. Los nuevos contenidos curriculares de los últimos años han dado lugar a un intenso proceso de desarrollo académico con la creación de departamentos de MG en 20 de las 36 facultades públicas de medicina del país. Conclusiones: La profesionalización académica de los trabajadores de MG/MF, así como la expansión de los cursos de formación profesional en esta especialidad, objetiva promover el interés de la MG/MF en los jóvenes médicos. Este desarrollo fortalece la investigación sobre MG/MF, lo que contribuye a mejorar la base institucional de la MG/MF como ciencia, lo que permite la interacción y colaboración más osada con otras ramas de la medicina y la real valorización de la MG/MF.

Como citar: Herrmann M, Giovanella L. Desenvolvimento e formação profissional em medicina geral e de família na Alemanha. Rev Bras Med Fam Comunidade. 2013;8(27):132-42. Disponivel em: http://dx.doi.org/10.5712/rbmfc8(27)679

\section{Palavras-chave:}

Medicina Geral

Medicina de Família e Comunidade Formação de Recursos Humanos Atenção Primária à Saúde

\section{Keywords:}

General Practice Family Medicine Human Resources Training Primary Health Care

Palabras clave: Medicina General Medicina Familiar y Comunitaria Formación de Recursos Humanos Atención Primaria de Salud 


\section{Introdução}

Nos países europeus, a atenção primária à saúde (APS) tem como profissional nuclear o médico generalista, que adquire diversas denominaçôes: médico geral e de família, general practitioner (GP), médico de família e comunidade ${ }^{1,2}$. Na Alemanha, a atenção por médico generalista, o Hausarzt, possui longa tradição, e a maioria da população adulta dispóe de um Hausarzt, que busca regularmente para a resolução de seus problemas de saúde. Nas últimas duas décadas, ao mesmo tempo em que foram desenvolvidas iniciativas para fortalecer a atenção primária na Alemanha, com implementação de programa de atenção centrada no médico generalista ${ }^{3}$, ocorreram importantes mudanças para a medicina geral e de família $\left(\mathrm{MGF}^{*}\right)$ : a) A presença acadêmica da MGF e suas estratégias de formação foram ampliadas, observando-se forte orientação em concepções fundamentadas em resultados e evidências; b) Ocorreu maior burocratização do trabalho; c) Surgiram novos modelos de atenção e concepçóes organizacionais, bem como a profissionalização de outros trabalhadores de saúde exigiu maior cooperação. Ainda assim, a atuação como médico generalista perdeu atratividade, principalmente no meio rural onde surgem impasses decorrentes de disparidades na distribuição regional de generalistas ${ }^{4}$.

O presente artigo tem como objetivo analisar o desenvolvimento profissional em medicina geral e de família (MGF) na Alemanha. Inicia com breve caracterização da estrutura do sistema de saúde e da prestação de serviços para contextualizar a atuação em medicina geral e de família na Alemanha e sua posição no sistema. Em seguida, descreve as características e os instrumentos de intervenção da MGF e apresenta as exigências para a especialização em MGF e o processo de qualificação. $\mathrm{Na}$ parte quatro, examina o desenvolvimento acadêmico da MGF naquele país e ao final discute desafios e perspectivas do desenvolvimento profissional em MGF na Alemanha.

\section{A estrutura ambulatorial e a prestação de serviços de medicina geral e de família na Alemanha}

Na Alemanha, a proteção social à saúde é garantida pelo Seguro Social de Doença (Gesetzliche Krankenversicherung-GKV) por meio de 145 Caixas. De afiliação compulsória, o Seguro Social de Doença alemão é financiado solidária e paritariamente por trabalhadores e empregadores, mediante contribuiçôes sociais proporcionais aos salários (taxa atual de $15,5 \%$ sendo $7,3 \%$ pagos pelo empregador e $8,2 \%$ pelo trabalhador) ${ }^{5}$. Garante ampla cobertura de serviços de saúde em todos os níveis conforme necessidades e cobre atualmente $90 \%$ da população. O financiamento da atenção à saúde na Alemanha é predominantemente público: $77 \%$ dos gastos em saúde são públicos e correspondem a $8,9 \%$ do PIB. Os gastos totais em saúde são elevados - em 2010 correspondiam a 11,6\% do PIB. A expectativa de vida é de 77,8 anos para homens e 82,8 anos para mulheres, levemente inferior à da França, Espanha, Itália ou Suécia ${ }^{6}$.

As Caixas do Seguro Social de Doença não prestam diretamente serviços de saúde. Para garantir atenção à saúde aos seus segurados as Caixas estabelecem contratos com diversos tipos de prestadores: associaçóes de profissionais de saúde, hospitais (governamentais, privados e filantrópicos) e farmácias privadas, para a assistência farmacêutica.

Para a atenção ambulatorial as Caixas estabelecem contratos com as Associações de Médicos Credenciados das Caixas, e esses médicos atendem os segurados como profissionais autônomos em seus consultórios privados (Praxis), em sua maioria individual (75\%).

A oferta das Caixas é ampla, sendo que a grande maioria (98\%) dos médicos em exercício em prática ambulatorial é credenciada pelas Caixas ( $85 \%$ credenciados e $13 \%$ como empregados) e somente $1,7 \%$ atua em prática privada exclusivamente ${ }^{7}$.

A remuneração dos profissionais pelas Caixas é realizada com base em casos tratados e limitada por tetos financeiros por consultório e especialidade. A atençáo ambulatorial é gratuita (em 2004 foi estabelecida uma taxa de copagamento de 10 euros por trimestre por médico procurado, que foi abolida em 2012).

As Associações de Médicos Credenciados das Caixas do Seguro Social de Doença ocupam posição-chave no sistema de saúde. São responsáveis pela garantia da atenção ambulatorial e coordenam as atividades dos outros prestadores de serviços, cujo acesso é dependente de seu encaminhamento. Essas associaçóes representam os médicos, estabelecem contratos coletivos com as Caixas e negociam o valor da remuneração setorial. Recebem os honorários de todos os médicos, que são separados

\footnotetext{
Medicina geral e de família é o equivalente a medicina de família e comunidade (Brasil e Espanha) ou Clínica Geral (Inglaterra).
} 
em um valor global para os médicos generalistas e outro para médicos especialistas, e os distribuem para cada médico, conforme o número de casos tratados e serviços prestados por trimestre.

As relaçôes financeiras na prestação de serviços médicos no setor ambulatorial na Alemanha, similares para médicos generalistas e especialistas, são ilustradas na Figura 1.

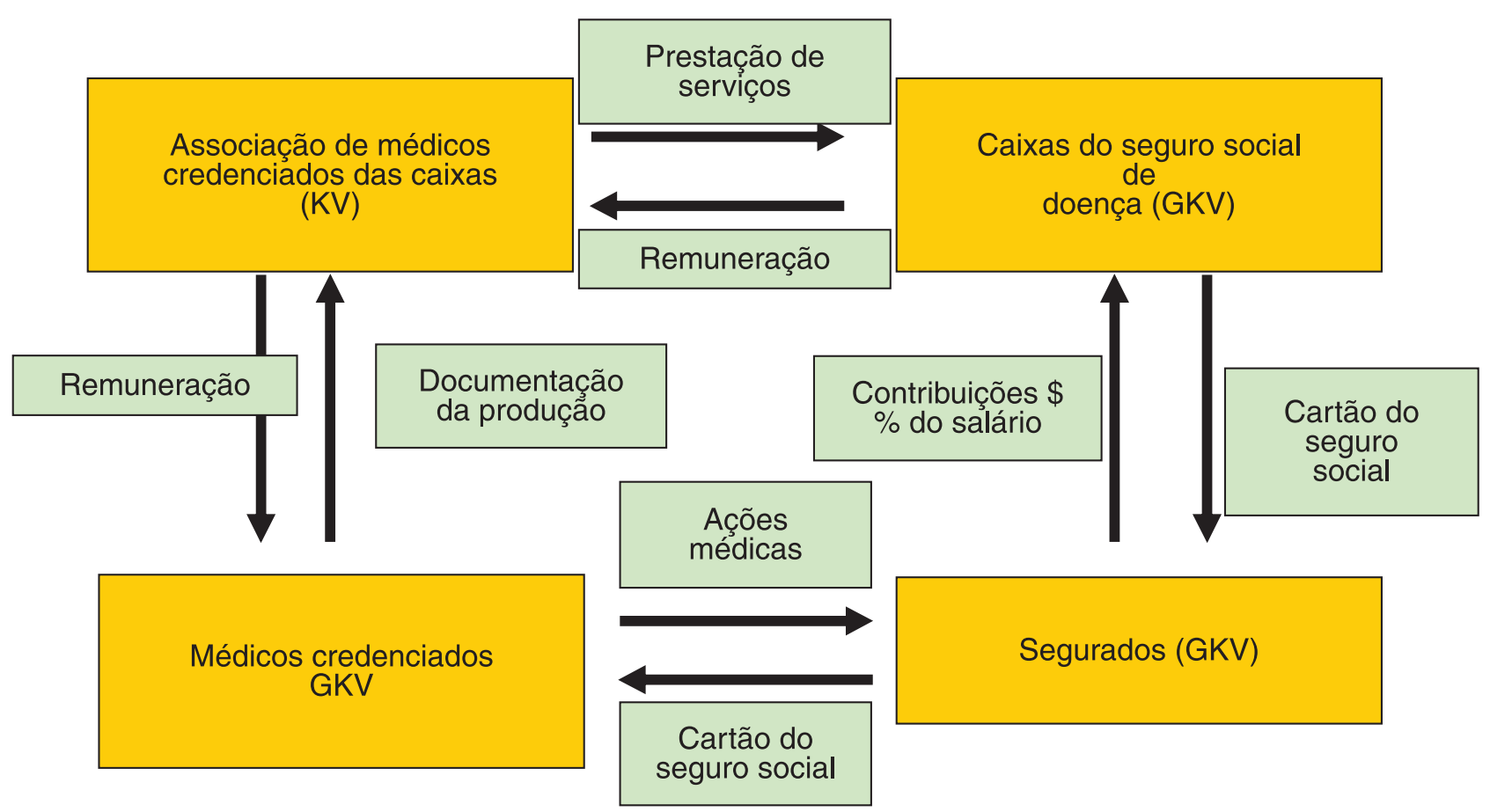

Figura 1. Relações financeiras na prestação de serviços médicos no setor ambulatorial na Alemanha. Fonte: elaborado pelos autores.

\section{A posição do médico generalista no sistema de saúde alemão}

Não há definição de um primeiro nível de atenção e uma porta de entrada preferencial. A atenção de primeiro contato pode ser prestada tanto pelo médico generalista quanto pelo médico especialista. Assim, o segurado do GKV, a cada atendimento, pode escolher qualquer médico credenciado, não sendo obrigatório o encaminhamento pelo generalista (Hausarzt), "médico da família/casa", denominação do generalista alemão, para consulta com especialistas. Ainda assim, em geral, o consultório médico do Hausarzt é o ponto de primeiro contato na busca por assistência médica e a maioria dos cidadáos refere ter um médico de família.

Nesse sentido, a posição da atenção primária no sistema de saúde é considerada fraca, pois o generalista não exerce a função de gatekeeper $^{8}$. Todavia, medidas para fortalecer a atenção pelos generalistas vêm sendo introduzidas. Desde 2007, ainda que com implementação lenta, todas as Caixas devem oferecer um programa de Atenção Centrada no Médico Generalista, que, voluntariamente escolhido pelo segurado, passa a exercer a função de coordenação dos cuidados ${ }^{3}$.

Existem diferenças significativas por toda a Europa na prática da medicina geral e familiar. Diversos países europeus consolidaram sistemas de saúde com base na atenção primária (Holanda, Reino Unido, Espanha, Irlanda, Itália, Portugal, Polônia, Letônia, Lituânia, Dinamarca, Noruega) centrada na atenção por médico generalista (general practitioner -GP). Nesses países, os usuários devem sempre primeiro procurar o seu GP, que os encaminha, se necessário, ao especialista, esta posição de gatekeeper lhes confere maior poder de decisão e uma maior importância na definição das trajetórias da atenção do que na Alemanha) ${ }^{2,9}$. Além disso, nesses países os GPs realizam muitas outras funções e procedimentos, que na Alemanha são exercidos por especialistas - como a prevenção ginecológica por ginecologistas ou o acompanhamento de hipertensos por cardiologistas. 
Os mais de 70 milhóes de segurados do Seguro Social de Doença alemão são assistidos no setor ambulatorial por 153.895 médicos e psicoterapeutas, inscritos na Associação Nacional de Médicos Credenciados das Caixas (KBV), o que corresponde a cerca de 2,2 médicos do setor ambulatorial por mil habitantes (considerado o total de médicos no país, a relaçáo é de 3,7 médicos por mil habitantes, quase o dobro da do Brasil, que é de 1,9 médico por mil habitantes). Entre os médicos credenciados 77.042 são médicos especialistas, 16.479 psicoterapeutas e 60.374 médicos generalistas (dados de 2009 $)^{10}$. Como médicos generalistas compreendem-se: especialistas em medicina geral e de família (cerca de 64\%), internistas, pediatras e médicos práticos.

O número total de médicos generalistas é considerado suficiente se relacionado com o planejamento de necessidades estabelecidos pelas Caixas, contudo observam-se importantes disparidades regionais na distribuição dos especialistas, com áreas desassistidas em regiôes rurais e na região leste da Alemanha ${ }^{4}$.

Esses mais de 60 mil médicos generalistas (Hausärzte, médicos do lar ou da família) incluem médicos atuantes na atenção generalista, contudo com distinta formação: especialistas em medicina geral e de família com formação específica (Allgemeinmedizin und Familienmedizin); internistas atuantes como generalistas; parte dos pediatras que também pode se inscrever como generalistas; e clínicos gerais sem formação específica, os médicos práticos (praktische Ärzte). Médicos práticos (ou simplesmente clínicos) é o termo profissional empregado para denominar médicos habilitados sem qualquer outra formação específica. Essa é uma categoria em extinção, pois vale somente para médicos já formados que eram licenciados nessa categoria após um determinado tempo de prática clínica generalista como assistentes. No âmbito da uniformização do direito europeu, com a implementação da resolução europeia Diretiva 93/16/CEE, a partir de 2003 não é mais possível se habilitar como médico generalista sem uma especialização adicional ${ }^{11}$.

Isso significa que hoje, para atuar como "especialista em medicina geral e de família”, é exigida qualificação mínima nos padrôes das diretrizes europeias, como será discutido na próxima seção.

\section{Características da medicina geral e de família na Alemanha}

Nos países da União Europeia, a formação especializada em MGF rege-se pela Diretiva UE 93/16, a qual define normas gerais, destinada a facilitar, sobretudo, a livre circulação de médicos entre países europeus e o reconhecimento mútuo de seus diplomas.

A Diretiva UE 93/16 regula a prática médica generalista ao definir critérios relativos ao acesso à formação especializada, à sua duração mínima, ao ensino e o lugar onde deve ser efetuada, bem como o controle a que deve ser submetida ${ }^{11}$. Em seu artigo 30 estabeleceu a obrigaçáo dos estados membros em criar uma formaçáo pós-graduada específica em medicina geral, de natureza mais prática do que teórica, com duração mínima de três anos, e desde 1998, de cinco anos. A formação prática deve ser realizada em serviços reconhecidos e credenciados, em meio hospitalar e em consultórios de medicina geral ou centros de saúde nos quais os médicos ministrem cuidados primários. A Diretiva UE 93/16 estipulou ainda que a incorporação de novos médicos no exercício da prática generalista a partir de 1995 deveria passar a depender da posse de um certificado de especialização adquirido conforme os critérios mínimos definidos de formação, sem prejuízo de direitos adquiridos dos generalistas já atuantes ${ }^{11}$.

A WONCA Europa avalia que, infelizmente, a diretiva coloca pouca ênfase no conteúdo e na qualidade da formação pós-graduada que municie os médicos e lhes confira as aptidóes necessárias para exercer a MGF em qualquer estado membro ${ }^{12}$.

$\mathrm{Na}$ Alemanha, a medicina geral e de família cumpre os critérios da Diretiva UE 93/16. É uma especialidade médica que tem como requisito cinco anos de formação prática, não acadêmica (nos moldes de uma residência médica), após os quais é necessário submeter-se a uma prova na Câmara Médica Estadual (órgão de regulação profissional correspondente aos Conselhos Federal e Regional de Medicina no Brasil).

$\mathrm{Na}$ Alemanha, de acordo com a Norma para a Formação Especializada da Câmara Médica Federal de 2003, o campo de atuação da medicina geral e de família (MGF) compreende a assistência médica generalista ao ser humano de qualquer faixa etária com acompanhamento ao longo da vida em qualquer manifestação de transtorno à saúde, considerando as dimensôes biológicas, psíquicas e sociais das enfermidades, sofrimento, problemas ou risco à saúde, bem como a competência para tomada de decisão sobre a inclusão de outros médicos e profissionais de saúde na atenção ${ }^{13}$. A MGF compreende a integração, centrada no paciente, da assistência médica, psíquica e social nos casos de doença. Engloba a atenção de casos agudos e 
o acompanhamento de portadores de enfermidades crônicas, a promoção e aconselhamento, a prevenção e diagnóstico precoce de enfermidades, a adoção de medidas de reabilitação, o trabalho em conjunto com todas as pessoas e instituiçôes importantes ao acompanhamento da saúde do paciente, o apoio a atividades comunitárias voltadas à promoçáo da saúde e o registro de todos os dados importantes para acompanhamento da condiçáo de saúde dos pacientes ${ }^{13}$.

A entidade científica da especialidade, a Sociedade Alemá de Medicina Geral e Familiar (Deutsche Gesellschaft für Allgemeinmedizin und Familienmedizin - DEGAM), na sua definição do campo de atuação da Sociedade Científica discerne o campo de trabalho, a forma de atuação, os princípios e missão da $\mathrm{MGF}^{14}$, conforme apresentado no Quadro 1.

Quadro 1. Definição da especialidade em medicina geral e de família na Alemanha.

\begin{tabular}{|l}
\hline Campo de trabalho \\
Forma de atuação \\
agudos e de longa duração, bem como nas áreas-chave de prevenção e reabilitação. \\
A forma de atenção do MGF engloba aspectos somáticos, psicossociais, socioculturais e ambientais. Na interpretação dos sintomas, achados \\
e enfermidades, é de particular importância que o conceito de doença, o ambiente e a história do paciente sejam considerados e apreciados \\
(compreensão hermenêutica dos casos). \\
A MGF tem como fundamentos uma relação estável de longo prazo entre médico e paciente e a anamnese vivenciada na ampla competência e \\
na continuidade do cuidado ao longo da vida. Os princípios do trabalho incluem a apreciação das características epidemiológicas do coletivo não \\
selecionado de pacientes com as consequentes condições especiais para a tomada de decisão coerente (esperar e manter o caso aberto sem \\
fechar o diagnóstico, vigiar e afastar casos de risco). \\
filtrar e conduzir de forma adequada e responsável, perante o paciente e a sociedade, as etapas do diagnóstico e da terapia, envolvendo outros \\
especialistas. \\
Missão \\
acompanhar e orientar o paciente no contexto de sua família e meio social, incluindo o ambiente doméstico (visita domiciliar). \\
realizar educação em saúde, especificamente na promoção de saúde de indivíduos, assim como da comunidade. \\
coordenar e integrar a atenção, por meio do encaminhamento específico a especialistas, a coordenação entre os diversos níveis de assistência, \\
a compilação e apreciação do conjunto de resultados e ações prestadas ao paciente por diferentes profissionais, contínua documentação e \\
avaliação dos resultados, bem como a intermediação de cuidados de enfermagem e outros apoios sociais ao paciente em seu meio.
\end{tabular}

Fonte: Degam (2002) ${ }^{14}$.

A MGF está fundamentada em três orientações: nosológica, perspectiva populacional e biográfica ideográfica centrada no paciente.

A atenção médica generalista passou nas últimas décadas por diversas transformaçôes, deixando de ser uma prática baseada no empirismo do Hausarzt de estilo tradicional para se transformar em uma especialidade em atenção primária. Segundo Gerlach ${ }^{15}$, a prática tradicional do Hausarzt caracterizava-se por: foco exclusivo nos " 5 sentidos", pesquisa empírica como fundamento de suas açóes, anamnese vivenciada, competências generalizadas (Quadro 2).

Hoje a medicina geral e de família na Alemanha modificou-se. Se por um lado restringe seu campo de atuação com a entrada de outros especialistas, como obstetras e pediatras, por outro amplia seus meios diagnósticos e incorpora novos procedimentos. Hoje as clínicas de médicos generalistas na Alemanha são em geral bem equipadas, prestando serviços de atenção individual de promoção, prevenção, diagnóstico e atenção a casos agudos e crônicos da população adulta (não atendem saúde da mulher e de crianças, que fica a cargo de ginecologistas, parteiras e pediatras, respectivamente). Dispóem de um conjunto de equipamentos e realizam diversos procedimentos na própria clínica, como ultrassonografia de tireoide e abdome, exame das funçóes cardíacas e circulatórias com eletrocardiograma, teste de esforço (ergometria), medição de pressão arterial de longa duração, exame de artérias periféricas e exames de função pulmonar (espirometria). Dispóem de um microlaboratório equipado para testes rápidos (em geral com fita e gota) para mensuração de glicose, colesterol, ureia, coagulação, exame de urina; realizam suturas e coletam material para exames.

Hoje a MGF se caracteriza por: envolvimento de especialistas, incorporação de novos procedimentos, uso de abordagens baseadas em evidências, análise do histórico clínico e das enfermidades detectadas e foco no paciente. 
Quadro 2. Características da prática tradicional do Hausarzt e do especialista em medicina geral e familiar ${ }^{15}$.

\section{Prática tradicional do Hausarzt}

Competências generalizadas: muitas vezes o Hausarzt era o único

médico na região e responsável pela assistência a todos os tipos de

problemas de saúde e todos os ciclos de vida, com prática clínica

abrangente (desde o berço até o túmulo).

0 foco exclusivo nos "5 sentidos": disponível 24 horas por dia, 0 Hausarzt tradicional atuava sem equipamento adicional, com base em seus cinco sentidos e nos instrumentos que cabiam nos bolsos do jaleco: 0 estetoscópio, o monitor de pressão arterial etc.

Pesquisa empírica como fundamento de suas ações: sua atuação baseava-se em sua experiência profissional e pesquisa empírica desenvolvida ao longo de muitos anos de prática. Havia muita dificuldade para transferir para a prática conhecimentos clínicos atualizados. Anamnese vivenciada: dispunha apenas de um histórico do paciente e do desenvolvimento dos quadros clínicos vivenciados ao longo do tempo de acompanhamento de gerações de uma mesma família.

Decisões hierárquicas: tomadas pelo médico, sem consulta aos pacientes

\section{Prática do especialista MGF}

Envolvimento de especialistas. 0 MGF se vê como aquele que envolve os especialistas e outras profissões da saúde em questões específicas. Deixa de reivindicar para si a função obstétrica ou o cuidado de crianças e a determinação de todos os valores laboratoriais em seu próprio consultório. Não precisa mais se responsabilizar por todas as emergências. Há serviços especializados para tal. Situa-se em um sistema cada vez mais fragmentado. Amplia os meios diagnósticos e incorpora novos procedimentos: as clínicas dos MGF são bem equipadas, prestando serviços de atenção individual de promoção, prevenção, diagnóstico e atenção a casos agudos e crônicos da população adulta

Uso de abordagens baseadas em evidências: como resultado das novas tecnologias de informação, amplamente disponíveis, hoje em dia, é possível recorrer rapidamente a conhecimentos sistematizados, e implementar abordagens baseadas em evidências como diretrizes clínicas e programas de gestão clínica estruturados.

Análise do histórico clínico e das enfermidades detectadas: a prática diária do médico geral e de família está fundamentada em informações coletadas e bem documentadas ao longo do processo de cuidado dos pacientes, e compartilhamento com os colegas de resultados devidamente documentados. A atuação padronizada domina a prática cotidiana dos médicos.

Foco no paciente: as decisões são compartilhadas. 0 MGF fomenta nos pacientes 0 comportamento de atores possuidores de ponto de vista crítico, participantes autônomos que podem colaborar ativamente para a melhora do quadro clínico (o MGF exerce o papel de companheiro, conselheiro)

Fonte: Sintetizado a partir de Gerlach (2002) ${ }^{15}$.

\section{Qualificação em medicina geral e de família na Alemanha}

A formação acadêmica em medicina na Alemanha é realizada em 36 faculdades de medicina públicas e gratuitas e apenas uma faculdade privada. A especialização em MGF ocorre ao final da formação médica básica de seis anos de faculdade, por meio de capacitação prática em serviços.

A formação pós-graduada em MGF tem duração de cinco anos (60 meses) em processo não acadêmico, no qual o candidato atua como médico assistente em formação em diversos serviços: consultórios de médicos generalistas, serviços secundários e terciários.

As regras de prática para a formação especializada têm alguma diferenciação nos estados, pois é regulamentada pelas câmaras médicas, similares aos nossos conselhos regionais de medicina. Deve contemplar entre 24 e 36 meses em medicina interna, como assistente em instituição hospitalar acreditada para a formação especializada, e 24 a 36 meses em ambulatório de medicina geral e familiar, com preceptoria de um especialista em $\mathrm{MGF}^{13}$.

Os 24 ou 36 meses em medicina interna são realizados no hospital, contudo podem incluir 18 meses no acompanhamento direto ao paciente no setor ambulatorial. Os 24 ou 36 meses de formação continuada na assistência ambulatorial em MGF são realizados como empregado em consultório de generalista, especialista em MGF.

Além disso, deve ser realizado um curso de no mínimo 80 horas de formação continuada no campo da assistência psicossomática básica ${ }^{13}$.

Uma importante dificuldade para a concretização dos cinco anos da formação especializada em serviço é que esta não está estruturada em um curso de residência contínuo. Cada médico assistente aspirante à especialidade deve procurar uma vaga e conseguir um contrato formal de trabalho temporário em um hospital e em um consultório de MGF responsável pela sua formação (preceptor).

A implementação da especialização está sob responsabilidade de cada Câmara Médica Estadual, que além de regular especificidades locais realiza as provas das especialidades. Em Berlim, por exemplo, foi definida a seguinte distribuição de 
práticas: um ano de cirurgia geral, um ano de medicina interna, um ano e meio de MGF, um ano livre à escolha do candidato (incluindo três meses em Anestesiologia) e 80 horas em atenção psicossomática. Após o fim da formação básica pode-se optar por formaçóes adicionais em tópicos específicos, como homeopatia, medicina desportiva, psicoterapia.

Ao fim dessa formação prática pós-graduada é prestado um exame na Câmara Médica Estadual. A cada cinco anos, o título deve ser revalidado. Para isso é necessário comprovar a participação em um determinado número de horas de seminários de formação continuada, junto ao respectivo conselho de medicina do estado.

O conteúdo normatizado pela Câmara Médica Federal da Alemanha dos conhecimentos, experiência, aprendizado e competências para formação especializada em medicina geral e de família inclui bases da medicina interna, da prática generalista e habilidades em métodos de diagnose e terapia (Quadro 3).

Quadro 3. Conteúdos programáticos da formação especializada em medicina geral e de família na Alemanha.

\begin{tabular}{|c|c|}
\hline Áreas & Conteúdos \\
\hline ledicina interna & $\begin{array}{l}\text { - aconselhamento em saúde, diagnóstico precoce de enfermidades, incluindo dependência química e violência, prevenção, introdução e realização de } \\
\text { medidas reabilitativas, bem como acompanhamento da convalescência } \\
\text { - detecção e tratamento de enfermidades não infecciosas, infecciosas, tóxicas e neoplásicas, imunológicas, doenças metabólicas, nutricionais e } \\
\text { degenerativas, inclusive as especificidades desses transtornos em idade avançada } \\
\text { - bases para o tratamento de tumores } \\
\text { - o acompanhamento médico paliativo } \\
\text { - a definição de indicações para provas diagnósticas, a coleta e o manuseio adequado de material para laboratório, bem como avaliação dos } \\
\text { resultados e correspondência ao respectivo quadro nosológico } \\
\text { - síndromes geriátricas e consequências de enfermidades em pacientes de idade avançada e farmacoterapia de idosos } \\
\text { - sintomas psicogênicos, reações psicossomáticas e interações psicossociais incluindo intervenções em momentos de crise, bem como as bases do } \\
\text { aconselhamento e tratamento de adictos } \\
\text { - medidas preventivas e de diagnóstico precoce } \\
\text { - transtornos nutricionais, incluindo terapias dietéticas, aconselhamento e treinamento } \\
\text { - realização e documentação do tratamento de diabetes } \\
\text { - as bases de enfermidades hereditárias, incluindo a indicação para aconselhamento em genética humana } \\
\text { - indicação e controle de medidas de fisioterapia } \\
\text { - as bases da terapia medicamentosa } \\
\text { - o tratamento emergências, incluindo medidas de socorro para manutenção e preservação de funções vitais e de reanimação } \\
\text { - avaliação das condições físicas, da capacidade de execução de atividades profissionais, bem como da necessidade de assistência e cuidado } \\
\text { prolongado em casos de incapacidades e dependência } \\
\text { - as bases da medicina intensivista }\end{array}$ \\
\hline $\begin{array}{l}\text { Outros conteúdos } \\
\text { da medicina geral } \\
\text { e de família }\end{array}$ & $\begin{array}{l}\text { - diagnóstico em medicina geral, aconselhamento e tratamento em todos os casos de transtornos da saúde e enfermidades em pacientes, em geral, } \\
\text { não selecionados } \\
\text { - a integração de ações médicas, psíquicas e sociais nos casos de doença } \\
\text { - acompanhamento e assistência médica e familiar ao longo da vida } \\
\text { - diagnóstico e tratamento coordenado de crianças e adolescentes portadores de síndromes comportamentais } \\
\text { - coordenação interdisciplinar, incluindo a introdução de outros métodos e apoios médicos, de cuidados de enfermagem e assistência social nas } \\
\text { concepções de tratamento e acompanhamento, principalmente em casos de pacientes multimórbidos } \\
\text { - tratamento de pacientes em seu meio familiar e domiciliar, em instituições prestadoras de cuidados continuados (ancionatos), bem como no seu meio } \\
\text { social, incluindo visitas domiciliares } \\
\text { - medidas promotoras de saúde, por exemplo, no âmbito de projetos comunitários } \\
\text { - medidas preventivas e de detecção precoce } \\
\text { - detecção de doenças associadas às dependências químicas, bem como execução de medidas específicas } \\
\text { - detecção, avaliação e solução de danos originários de influências do meio ambiente e do meio social, incluindo influências do meio de trabalho } \\
\text { - tratamento de enfermidades do aparelho esquelético e muscular com especial consideração de distúrbios funcionais } \\
\text { - técnicas necessárias para a atenção em medicina geral e familiar para o tratamento e cuidado de ferimentos, incisão, extração, extirpação e excisão } \\
\text { para a coleta de material para análises laboratoriais, inclusive com aplicação de anestesia local e periférica }\end{array}$ \\
\hline \begin{tabular}{|l} 
Métodos \\
diagnósticos e \\
terapêuticos em \\
medicina interna
\end{tabular} & $\begin{array}{l}\text { - electrocardiograma } \\
\text { - ergometria } \\
\text { - eletrocardiograma de longa duração } \\
\text { - medições de pressão arterial de longa duração } \\
\text { - análise espirométrica das funções pulmonares } \\
\text { - ultrassonografia do abdome e retroperitônio, incluindo os aparelhos genital e urinário } \\
\text { - ultrassonografia da glândula tireoide } \\
\text { - sonografia-doppler das extremidades e dos vasos extracranianos } \\
\text { - técnicas de punção e de cateterismo, incluindo a aquisição de material para laboratório } \\
\text { - infusão, transfusão e terapias substitutivas à infusão de sangue, alimentação enteral e parenteral } \\
\text { - proctoscopia }\end{array}$ \\
\hline
\end{tabular}

Fonte: Bäk (2003) $)^{13}$. 


\section{Desenvolvimento acadêmico da MGF na Alemanha}

A profissionalização da medicina na Alemanha se consolidou com a normatização instituída, em 1852, que estabeleceu a obrigatoriedade de curso superior para o exercício da medicina. O estabelecimento da medicina geral e de família como disciplina acadêmica, todavia, ocorreu lentamente, somente a partir da segunda metade do século XX, como apresentado no Quadro $4^{16}$.

Quadro 4. Passos do desenvolvimento acadêmico da medicina geral e de família na Alemanha.

\begin{tabular}{|ll|}
$\mathbf{1 9 2 5}$ & Circulação do primeiro periódico na área, Der Landarzt (O médico rural), que deu origem ao atual Zeitschrift für Allgemeinmedizin (Revista de Medicina Geral) \\
$\mathbf{1 9 6 6}$ & Primeiro contrato de docente em medicina geral e de família (Universidade de Freiburg) \\
$\mathbf{1 9 6 6}$ & Fundação da Sociedade Alemã de Medicina Geral e de Família \\
$\mathbf{1 9 7 2}$ & Decisão do Conselho Nacional de Medicina para a implementação de institutos de medicina geral nas faculdades de medicina \\
$\mathbf{1 9 7 6}$ & Primeira cadeira de MGF \\
$\mathbf{1 9 7 7}$ & Primeiros professores titulares em MGF \\
$\mathbf{1 9 7 8}$ & Primeira disciplina obrigatória de medicina geral nas faculdades de medicina: "Introdução à prática médica generalista" \\
$\mathbf{1 9 9 9}$ & Nove professores titulares em MGF \\
$\mathbf{1 9 9 9}$ & Parecer do Conselho Científico Nacional Alemão: "Perspectivas da disciplina medicina geral nas universidades" \\
$\mathbf{1 9 9 9}$ & Diretrizes para promover a pesquisa em MGF nas faculdades da medicina \\
$\mathbf{2 0 0 4}$ & Novo Regulamento Nacional para Licença do Exercício da Medicina (Approbationsordnung) inclui um estágio e um curso obrigatórios em MGF no quarto ano \\
$\mathbf{2 0 1 2}$ & Revisão do regulamento nacional para licença do exercício da medicina: define como obrigatório um estágio prático de um mês em consultório de MGF \\
\hline
\end{tabular}

Fonte: Adaptado de Gerlach (2010) ${ }^{16}$.

A formação médica nos últimos anos passou a incluir novos módulos em medicina geral e de família e períodos de prática no consultório do generalista, bem como a MGF estabeleceu bases acadêmicas, com a criaçáo de departamentos/institutos de MGF na maior parte das faculdades de medicina.

Atualmente, o curso de medicina deve incluir pelo menos uma disciplina obrigatória em medicina geral e de família e um período de prática mínimo de duas semanas em consultório de generalista, no quarto ano. A partir de 2012, é obrigatório também no sexto ano, durante o internato, um estágio prático de um mês em consultório de MGF. Além disso, o aluno pode escolher durante o internato fazer um bloco prático de quatro meses em consultório do generalista, assim como foi definida uma regra para aumento progressivo da oferta pelas universidades de possibilidades de práticas em consultório generalista, que deve alcançar $100 \%$ em $2019^{17}$.

Para a realização de estágios práticos, os departamentos de MGF credenciam consultórios de especialistas em medicina geral e de família que atuam como preceptores, recebem uma formaçáo continuada e pequena remuneraçáo por dia de estágio (25 euros). Nacionalmente, existem cerca de cinco mil consultórios MGF credenciados para formação acadêmica prática (entre 42 e 350 por faculdade) ${ }^{18}$.

Atualmente, em 20 das 37 faculdades de medicina há professor titular em MGF e departamento/instituto de MGF, que além da docência realiza pesquisas na área, sendo que 14 departamentos concorreram em editais e receberam financiamento externo para pesquisa ${ }^{18}$. Nacionalmente, há cerca de 500 profissionais com contratos docentes em MGF. A tendência é de criaçáo de departamentos MGF em todas as faculdades, ainda que em algumas faculdades permaneçam docentes apenas com contratos temporários e estrutura incipiente.

Em comparação internacional, contudo, o desenvolvimento acadêmico na Alemanha ainda é baixo e foi mais lento do que em países com atenção primária por médico generalista tradicionalmente forte, como na Holanda, Reino Unido e países escandinavos, nos quais o maior reconhecimento da assistência prestada pelo MGF à populaçáo foi acompanhado de esforços para intensificar a formação, a educação continuada e o fomento da pesquisa em MGF. Como apresentado por Gerlach ${ }^{16}$, na Holanda, Reino Unido e países escandinavos a institucionalização universitária da MGF já foi concluída. Nesses países, todas as faculdades de medicina têm um departamento de MGF, enquanto na Alemanha somente em cerca da metade das faculdades de medicina há departamento específico, e na França e na Itália, por outro lado, não há praticamente iniciativa universitária em MGF. Na Holanda e Reino Unido a formação especializada em MGF também está ligada à universidade, e nos países escandinavos são oferecidos mestrados para aprofundamento do conhecimento e competências em pesquisa em $\mathrm{MGF}^{19}$. 


\section{Desafios da medicina geral e de família na Alemanha}

O processo de profissionalização e academização da MGF e a ampliação da formação na graduação visa valorizar a especialidade e aumentar a atratividade da MGF para os jovens médicos. Um dos desafios a enfrentar é a evolução insuficiente no número de médicos generalistas, especialmente em áreas rurais e na regiáo leste, observando-se um processo de envelhecimento da categoria.

Em comparação com a evolução do número total de médicos na Alemanha ocorreu redução da proporção de generalistas nos últimos anos. Enquanto em 1979 65\% dos médicos do setor ambulatorial atuavam como generalistas, em 2003 essa proporção passara para 50\%, e em 2009 atuavam como generalistas $48 \%$ do médicos do setor ambulatorial ${ }^{10}$. A estimativa é que essa tendência se acentue, caso não sejam implementados incentivos adequados, pois observou-se reduçấo do número de provas realizadas para a especialidade MGF (2007 = 1.938 provas; $2009=1.168$ provas $)$ e do número de novos consultórios abertos na especialidade $(2007=1.189 ; 2009=930)$; ademais do envelhecimento da categoria: $66 \%$ dos generalistas têm mais de 50 anos e $20 \%$ mais de 60 anos de idade.

Os principais motivos para a perda da atratividade da MGF na Alemanha, em comparação com outras especialidades médicas, são as dificuldades para treinamento na residência, já mencionadas, o menor status social e econômico do profissional, os riscos financeiros no caso de bancar um consultório, as exigências profissionais do ramo na abordagem biopsicossocial e as condiçóes de trabalho, especialmente exigências de elevadas jornadas de trabalho que a atuação em MGF impóe ${ }^{20}$.

A carga de trabalho de um generalista em seu consultório na Alemanha é elevada. Trabalham mais de 50 horas semanais, atendendo em média 243 pacientes por semana, com duraçáo de consultas em torno de 7,8 minutos ${ }^{21}$. O número de contatos médicos é excessivo, estimado em 18 contatos por habitante/ano ${ }^{22}$. Recente avaliação de consumidores do Euro Health Consumer Index (EHCI) posicionou a Alemanha em 140 lugar entre 34 países europeus em 2012, enquanto em 2009 ocupava a sexta posição ${ }^{23}$.

Além disso, mudanças na atuação profissional e no contexto político social contemporâneo comprometem a autonomia profissional, levando à insatisfação. Cada vez mais os médicos gerais e familiares são forçados a considerar aspectos de natureza jurídica ou econômica na tomada de decisão, e atividades administrativas e organizacionais passam a ocupar a maior parte do tempo. Além disso, mudanças de valores sociais - que se expressam, por exemplo, na relação médico-paciente, com maior amparo legal, pacientes mais informados e exigentes por tomada de decisão compartilhada - questionam a autonomia e a relação hierárquica tradicional da atuação do MGF.

A crescente especialização da medicina significa concorrência entre as diversas disciplinas médicas e o risco de apropriação de campos pertinentes à MGF. Ao mesmo tempo, a profissionalização acadêmica de outros trabalhadores da saúde, que na Alemanha tradicionalmente são apenas formaçôes técnicas, não universitárias (fisioterapia, enfermagem, fonoaudiologia...), representa outro campo de conflitos. A extensão das competências atribuídas às outras profissóes traz consigo novas exigências de cooperação entre os diversos profissionais de saúde que são (infelizmente) percebidas como uma ameaça à autonomia profissional do médico. Novos modelos de atenção que incorporam outros profissionais autônomos como enfermeiras comunitárias (Agnes) com delegação de funçôes, desenvolvidos para assegurar a prestação de assistência médica em regiôes rurais, apoiar as atividades de médicos gerais e familiares e aliviar sua carga de trabalho, são confrontados com posicionamentos críticos, pois os MGF temem eventual substituição de campos de atuação.

\section{Considerações finais: perspectivas para o desenvolvimento profissional da MGF na Alemanha}

Neste contexto, face às mudanças sociais, a MGF na Alemanha encontra-se sob uma "dupla pressão de desenvolvimento e adaptação". Por um lado, deve adaptar-se quantitativa e qualitativamente às transformações sociais enquanto, por outro lado, a profissão deve confrontar-se e lidar com a questão da legitimidade e da atuação autônoma para fortalecer sua posição no sistema de saúde.

As transformações demográficas e sociais com envelhecimento populacional e fragmentação do sistema de atenção e, principalmente, a crescente dificuldade em garantir uma atenção médica primária abrangente exigem o fortalecimento da 
MGF e funcionam, assim, como propulsoras do desenvolvimento acadêmico da disciplina. O futuro exercício da função de médico generalista e de família requer a integração de competências dialógicas para cooperação entre os diversos ramos profissionais da saúde que se encontram em fase de profissionalização e tomada de decisão em conjunto com o paciente.

O escritório europeu da OMS recomenda amplos esforços, inclusive financeiros, para a criação de departamentos acadêmicos em MGF em todas as faculdades de medicina. Considera imprescindível o reconhecimento da MGF como cadeira acadêmica e campo específico, para que seja legitimada como parceira ampla na assistência médica ${ }^{24}$. A experiência alemã mostra a possibilidade de uma academização rápida da MGF a partir da normatização de requerimentos curriculares na disciplina, com a criação de departamentos de MGF na maior parte das faculdades, que se carece no Brasil.

Simultaneamente, o fomento do desenvolvimento profissional acadêmico fortifica a pesquisa genuína praticada no campo da MGF. Uma medicina geral e familiar de base institucional aprimorada oferece, como ciência, possibilidades de interação e colaboração mais arrojadas com outros ramos da medicina e a real valorização da MGF.

\section{Agradecimentos}

À Coordenação de Aperfeiçoamento de Pessoal de Nível Superior (Capes) (www.capes.gov.br/) e ao Serviço Alemão de Intercâmbio Acadêmico / Deutschem Akademischen Austauschdienst (DAAD) pelo apoio na visita científica de curta duração de Markus Herrmann ao Brasil e estágio sênior no exterior de Ligia Giovanella, processo Capes n. BEX 3831/11-6.

\section{Referências}

1. Saltman RB, Rico A, Boerma WGW. Primary care in the driver's seat? Organizational reform in European primary care. Berkshire: Open University Press; 2006.

2. Giovanella L. A atenção primária à saúde nos países da União Europeia: configurações e reformas organizacionais na década de 1990. Cad Saúde Pública. 2006; 22(5): 951-964. http://dx.doi.org/10.1590/S0102-311X2006000500008

3. Giovanella L. Redes integradas, programas de gestão clínica e generalista coordenador: análise das reformas recentes do setor ambulatorial na Alemanha. Ciênc Saúde Coletiva. 2011; 16(supl.1): 1081-1096.

4. Greß S, Stegmüller K. Gesundheitliche Versorgung in Stadt und Land - Ein Zukunftskonzept. Bonn: Friedrich-Ebert-Stiftung; 2011.

5. Reiners H, Müller O. Die Reformfibel. Handbuch der Gesundheitsreformen. Berlin: KomPart-Verlag; 2012.

6. Organisation for Economic Co-operation and Development - OECD. Health at a Glance: Europe; 2012. OECD Publishing. http://dx.doi. org/10.1787/9789264183896-en

7. Bundesärztekammer. Arbeitsgemeinschaft der deutschen Ärztekammern. Tätigkeitsbericht 2010 der Bundesärztekammer. Berlin: Bundesärztekammer; 2011.

8. Macinko J, Starfield B, Shi L. The Contribution of Primary Care Systems to Health Outcomes within Organization for Economic Cooperation and Development (OECD) Countries, 1970-1998. Health Serv Res. 2003; 38:831-65. http://dx.doi.org/10.1111/1475-6773.00149

9. Herrmann M, Braun U, Schwantes U. Stärkung der häuslichen Versorgung durch ein Primärarztsystem. Jahrb Kritische Med. Bd. 32. 2000; 38-57.

10. Kassenärztliche Bundesvereinigung - KBV. Fakten und Zahlen. Bonn: KBV; 2012. [acesso em 2012 Dez. 01]. Disponível em: http://www.kbv.de/24854.html.

11. União Europeia - UE. Comunidade Económica Europeia - CEE. Directiva 93/16/, de 05 de abril de 1993. Destinada a facilitar a livre circulação dos médicos e o reconhecimento mútuo dos seus diplomas, certificados e outros títulos. Jornal Oficial 165, 07 jul. 1993. p. 1. [acesso em 2012 Dez. 04]. Disponível em: http://eur-lex.europa.eu/LexUriServ/LexUriServ.do?uri=CONSLEG:1993L0016:20070101:PT:PDF.

12. World Organization of National Colleges, Academies - WONCA Europa. A Definição Europeia de Medicina Geral e Familiar (Clínica Geral / Medicina Familiar). Barcelona: WONCA; 2002. p. 6-8. [acesso em 2012 Dez. 01]. Disponível em: http://www.woncaeurope.org/sites/default/files/documents/ European\%20Definition\%20in\%20Portuguese.pdf.

13. Bundesärztekammer - BÄK. Musterweiterbildungsordnung Bundesärztekammer 2003 (MWBO 2003. Stand 25.06.2010. Bundesärztekammer; 2003. [acesso em 2012 Dez. 01]. Disponível em: http://www.bundesaerztekammer.de/downloads/MWBO_25062010-2.pdf.

14. Deutsche Gesellschaft für Allgemeinmedizin und Familienmedizin - DEGAM. Fachdefinition: Beschluss der Jahreshauptversammlung vom 21.9.2002. DEGAM; 2002. [acesso em 2012 Dez. 01]. Disponível em: http://www.degam.de/index.php?id=303.

15. Gerlach FM. Allgemeinmedizin in einem modernen Gesundheitssystem - Konsequenzen für Forschung, Lehre und Praxis (Teil 1), Z. ärztl. Fortbild Qual sich. 2002; 96: 243-249. 
16. Gerlach FM. Allgemeinmedizin als wissenschaftliches Fach. Stand und Perspektiven. apresentacao. Homburg: Institut für Allgemeinmedizin Frankfurt 2010. [acesso em 2012 Dez. 04]. Disponível em: http://www.allgemeinmedizin.uni-frankfurt.de/aktuelles/2010/gerlach-Allgemeinmedizin_ als_wissenschaftliches\%20_Fach_20052010.pdf.

17. Gerlach FM. Comunicação pessoal ao autor em 27.11.2012 no Institut für Allgemein Medizin JW Goethe Universität Frankfurt.

18. Baum E, Ehrhardt M. Sektionsbericht Studium und Hochschule 2010. Z Allgemeinmedizin. 2010; 86(9): 345-8.

19. Herrmann M, Lichte T, Engeser P, Wächtler H. Akademische Professionalisierung (in) der Allgemeinmedizin. Erfahrungen, Bewertungen, Ausblick. Z. Allg. Med. 2003; 79: 609-612.

20. Herrmann M, Lehmann B. Professionsentwicklung der Allgemeinmedizin. In Dick M., Korfmacher S, Mieg H, Marotzki W. (Hrsg): Handbuch Professionsentwicklung. Huber; 2013. No prelo.

21. Koch K, Gehrmann U, Sawicki PT. Primärärztliche Versorgung in Deutschland im internationalen Vergleich. Ergebnisse einer strukturvalidierten Ärztebefragung. Deutsch Ärzteblat. 2007; 104(38):A 2584-91.

22. Schlenker RU, Schwartz FW, Grobe TG, Behrens K. Barmer GEK Arztreport. Schriftenreihe zur Gesundheitsanalyse Band 1; Berlin: Barmer GEK; 2010. [acesso em 2012 Dez. 12]. Disponível em: http://www.barmer-gek.de/barmer/web/Portale/Presseportal/Subportal/Infothek/Studien-und-Reports/ Arztreport/Arztreport-2010/PDF-Pressemappe,property=Data.pdf

23. Health Consumer Powerhouse. Euro Health Consumer Index 2012 Report. [acesso em 2013 Abr. 02]. Disponivel em: http://www.healthpowerhouse. com/files/Report-EHCl-2012.

24. World Health Organization - WHO. Framework for professional and administrative development of general practice / family medicine in Europe. EUR/ ICP/DLVR 0401 01. Copenhagen: WHO Regional Office for Europe; 1998. 